\title{
Piccolo genotype modulates neural correlates of emotion processing but not executive functioning
}

\author{
S Woudstra ${ }^{1,2,3,4,5}$, Z Bochdanovits ${ }^{2,5}$, M-J van Tol ${ }^{6,7}$, DJ Veltman ${ }^{1,5}$, FG Zitman ${ }^{3}$, MA van Buchem ${ }^{4,8}$, NJ van der Wee ${ }^{3,4}$, \\ EM Opmeer ${ }^{6}$, LR Demenescu, ${ }^{6,9}$, A Aleman ${ }^{6,10}$, BW Penninx ${ }^{1,3,5,6}$ and WJ Hoogendijk $k^{1,5,11}$
}

Major depressive disorder (MDD) is characterized by affective symptoms and cognitive impairments, which have been associated with changes in limbic and prefrontal activity as well as with monoaminergic neurotransmission. A genome-wide association study implicated the polymorphism rs2522833 in the piccolo (PCLO) gene-involved in monoaminergic neurotransmission-as a risk factor for MDD. However, the role of the PCLO risk allele in emotion processing and executive function or its effect on their neural substrate has never been studied. We used functional magnetic resonance imaging (fMRI) to investigate PCLO risk allele carriers vs noncarriers during an emotional face processing task and a visuospatial planning task in 159 current MDD patients and healthy controls. In PCLO risk allele carriers, we found increased activity in the left amygdala during processing of angry and sad faces compared with noncarriers, independent of psychopathological status. During processing of fearful faces, the PCLO risk allele was associated with increased amygdala activation in MDD patients only. During the visuospatial planning task, we found no genotype effect on performance or on BOLD signal in our predefined areas as a function of increasing task load. The PCLO risk allele was found to be specifically associated with altered emotion processing, but not with executive dysfunction. Moreover, the PCLO risk allele appears to modulate amygdala function during fearful facial processing in MDD and may constitute a possible link between genotype and susceptibility for depression via altered processing of fearful stimuli. The current results may therefore aid in better understanding underlying neurobiological mechanisms in MDD. Translational Psychiatry (2012) 2, e99; doi:10.1038/tp.2012.29; published online 3 April 2012

\section{Introduction}

Major depressive disorder (MDD) is a common, multifactorial disorder and is one of the leading causes of years lost due to disability. ${ }^{1,2}$ Its core symptoms are affective, manifesting themselves in depressed mood accompanied by low self-esteem and loss of interest in normally enjoyable activities. Other MDD symptoms include cognitive impairments, such as concentration and planning problems. ${ }^{3}$ The presence and severity of both affective and cognitive symptoms can be evaluated by neuropsychological assessments, ${ }^{4}$ and may be reflected in altered regional brain activity as measured using fMRI. ${ }^{5-7}$

Twin studies have shown that up to $40 \%$ of MDD is genetically determined. ${ }^{8}$ Much of the candidate gene literature has focused on genes from the monoaminergic neurotransmitter system, such as the serotonin transporter, monoamine oxidase $A$ and tryptophan hydroxylase $2 .^{9-14}$ A recent study by our consortium demonstrated that candidate genetic association studies are not well replicated, ${ }^{15}$ which suggests that a hypothesis-free approach is more useful to identify possible genetic variants that contribute to MDD. Previously, we conducted a genome-wide association study (GWAS) in MDD using $\sim 500000$ singlenucleotide polymorphisms (SNPs), showing one SNP (rs2522833) located at position 82453708 (hapmap genome build 37.1 ) in the piccolo gene (PCLO) in particular to be of interest in the genetic model for MDD. ${ }^{16}$ This association was replicated in some studies, ${ }^{16-18}$ but not in others. ${ }^{19,20}$ The rs2522833 SNP changes the hydrophilic, uncharged amino acid serine to the charged amino acid alanine in the calciumbinding $\mathrm{C} 2 \mathrm{~A}$ domain of PCLO and may affect protein stability. ${ }^{21}$ The PCLO protein is localized at the cytomatrix of the presynaptic active zone and is important in monoaminergic neurotransmission in the brain. ${ }^{16,22}$ Recently, Schuhmacher et al. ${ }^{23}$ showed that the PCLO SNP rs2522833 is associated with antidepressant treatment response, supporting the involvement of PCLO in MDD. The mechanism through which this PCLO risk allele contributes to MDD is not known, but its primary role in monoaminergic neurotransmission suggests that it may affect activity of brain circuitry involved in emotional information processing and/or executive functioning in MDD. ${ }^{24}$

\footnotetext{
${ }^{1}$ Department of Psychiatry, VU University Medical Center, Amsterdam, The Netherlands; ${ }^{2}$ Department of Medical Genomics, VU University Medical Center, Amsterdam, The Netherlands; ${ }^{3}$ Department of Psychiatry, Leiden University Medical Center, Leiden, The Netherlands; ${ }^{4}$ Leiden Institute for Brain and Cognition, Leiden University, Leiden, The Netherlands; ${ }^{5}$ Neuroscience Campus Amsterdam, VU University, Amsterdam, The Netherlands; ${ }^{6}$ BCN Neuroimaging Center and Department of Neuroscience, University Medical Center Groningen, University of Groningen, Groningen, The Netherlands; ${ }^{7}$ Leibniz Institute for Neurobiology, Otto von Guericke University, Magdeburg, Germany; ${ }^{8}$ Department of Radiology, Leiden University Medical Center, Leiden, The Netherlands; ${ }^{9}$ Department of Psychiatry and Psychotherapy, RWTH Aachen University, Aachen, Germany; ${ }^{10}$ Department of Psychology, University of Groningen, Groningen, The Netherlands and ${ }^{11}$ Department of Psychiatry, Erasmus MC, Rotterdam, The Netherlands Correspondence: S Woudstra, Department of Psychiatry, MF-A422, VU University Medical Center, PO Box 7057, Amsterdam 1007 MB, The Netherlands.
} E-mail: s.woudstra@ vumc.nl

Keywords: emotion processing; executive function; genome-wide association; major depressive disorder; neuroimaging genetics; PCLO

Received 20 December 2011; revised 15 February 2012; accepted 3 March 2012 
Abnormal emotion processing of, for example, facial stimuli is one of the intermediate phenotypes that can be considered closer to the neurobiological substrate of MDD than the clinical diagnosis itself. ${ }^{6}$ Identification of emotional facial expressions is reportedly impaired in MDD, ${ }^{25}$ and functional imaging studies have shown aberrant activity of emotional face processing related areas, including the amygdala, striatum, insula, parahippocampal area and dorsolateral prefrontal cortex (PFC), using an emotional faces task in healthy controls $(\mathrm{HC}) .{ }^{26}$ It is thought that serotonin has an important role in emotional face processing and dysregulation of the serotonergic system is associated with altered amygdala activity as well as the occurrence of MDD. 9,27,28

Another intermediate phenotype for MDD may be executive dysfunction, including impaired working memory and planning/problem solving. Previous studies in MDD have shown abnormal task performance and increased activity in taskrelated brain areas, ${ }^{7,29,30}$ using the Tower of London visuospatial planning task (ToL) and the n-back working memory task. $^{31-34}$ Apart from the PFC, other areas involved in planning include the striatum, and a visuospatial processing system, including the precuneus and inferior parietal cortex. ${ }^{29,34}$ The association of executive function with monoaminergic neurotransmission is thought to be bidirectional: PFC function is shaped by monoaminergic neurotransmitters, but ascending modulatory monoaminergic systems may also be affected by the PFC. ${ }^{24}$ Studies suggests that the PFC is able to recruit monoamine systems in specific circumstances, for example, to optimize the coping strategy for stress. ${ }^{35,36}$

Over the last few years, imaging genetics has shown to be a powerful tool in unraveling neurobiological pathways in various psychiatric disorders. However, to date, studies in MDD have been rare, ${ }^{10,37,38}$ and the role of the $P C L O$ risk allele on emotional information processing and executive function has never been studied. Given that PCLO is associated with MDD, we hypothesized that the PCLO risk allele-relative to the nonrisk allele-is associated with similar changes in activity in emotional processing and in executive function related areas as described for MDD. In addition, we aimed to investigate the relationship of PCLO with current psychopathology during emotional face processing and executive function.

\section{Materials and methods}

Subjects. A sample of 159 subjects participating in the Netherlands Study for Depression and Anxiety (NESDA) was selected to take part in this genetic neuroimaging study. Details of the NESDA study are extensively described elsewhere. ${ }^{39}$ Genotype data were collected through the genetic association information network (GAIN) study for MDD, in which 1862 cases and 1857 controls were genotyped. ${ }^{40}$ Magnetic Resonance Imaging (MRI) data were available from the MRI NESDA study, in which 301 subjects aged between 18 and 57 years participated. ${ }^{41}$ During a 1-h protocol, subjects underwent MR imaging while performing different tasks, including the ToL paradigm, the emotional faces paradigm and a verbal memory task, structural imaging and resting state imaging.
Exclusion criteria were: presence of MRI contraindications, DSM-IV axis I disorder other than MDD, Panic Disorder or social anxiety disorder (except generalized anxiety disorder) lifetime, or any DSM-IV disorder (for $\mathrm{HC}$ ), dependence or recent abuse of alcohol and/or drugs, hypertension, major internal and/or neurological disorders and use of psychotropic medication (other than stable use of a selective serotonin reuptake inhibitor (SSRI) or incidental use of benzodiazepines). To assess depressive symptom characteristics and severity scores, the Inventory of Depressive Symptomatol$\mathrm{ogy}^{42}$ and the Montgomery-Åsberg Depression Rating Scale, ${ }^{43}$ were used. For the present report, we included only subjects that met the 6-month recent DSM-IV criteria for current MDD, or no lifetime DSM-IV diagnosis. Owing to missing genotype data, technical problems during scanning and/or insufficient task performance, our final sample for this study consisted of 126 subjects for the emotional face processing study and 159 subjects for the executive function study (see Supplementary Table 1 for an overview). All participants provided written informed consent and the study was approved by the Ethics Committees at the VU University Medical Center, Amsterdam, the Leiden University Medical Center and the University Medical Center Groningen.

Demographics. The 126 subjects in the emotional faces study included 91 PCLO risk allele carriers (PCLO +; 25 homozygous and 66 heterozygous for the 'C' risk allele) and 35 homozygous ' $A$ ' nonrisk allele carriers (PCLO-). The ratio of PCLO + and PCLO- in the executive function study did not significantly differ (110 PCLO + ; 33 homozygous and 77 heterozygous for the ' $C$ ' risk allele, and 49 homozygous ' $A$ ' nonrisk allele carriers; PCLO-) from the ratio in the emotional faces study $\left(\chi^{2}[\mathrm{df} 1]=0.31 ; P>0.05\right)$. For the analyses, we formed two groups: one group consisting of subjects carrying the risk allele, and one group consisting of subjects not carrying the risk allele. From this point forward, we will refer to these groups as PCLO + and PCLO-, respectively. PCLO groups did not differ with regard to age, gender, education, MDD/HC ratio, depression severity or SSRI use/duration (see Table 1). Thirty-four individuals were using SSRIs, and 15 individuals were using nonpsychotropic medication other than SSRIs. All subjects reported Western European ancestry.

Genotyping. Genotyping for the genetic association information network study was performed by Perlegen and is described in detail elsewhere. ${ }^{16}$ Observed genotypes in our sample did not deviate from Hardy-Weinberg equilibrium (emotional face processing paradigm): $\chi^{2}$ [df 2] $=0.37$; $P>0.05$; CC: AC: $\mathrm{AA}=35: 66: 25 ; P>0.05$; executive functioning paradigm: $\chi^{2}$ [df 2] $=0.07$; CC: $A C: A A=33: 77: 49$; $P>0.05$; http://www.oege.org/software/hardy-weinberg.html).

Emotional information processing paradigm. An emotional faces task, based on the event-related fMRI paradigm used by Wolfensberger et al. ${ }^{44}$ was used in the present study and has been extensively described elsewhere. ${ }^{5}$ Briefly, 24 stimuli-selected from the Karolinska Directed Emotional 
Table 1 Demographic characteristics of total sample

\begin{tabular}{|c|c|c|c|c|c|c|c|c|c|c|}
\hline \multirow[t]{2}{*}{ Characteristics } & \multicolumn{5}{|c|}{ Tower of London planning task } & \multicolumn{5}{|c|}{ Emotional face paradigm } \\
\hline & Total & $\begin{array}{c}\text { Genotype } \\
\text { PCLO+ }\end{array}$ & PCLO- & $\chi^{2}$ & P-value & Total & $\begin{array}{c}\text { Genotype } \\
\text { PCLO+ }\end{array}$ & PCLO- & $\chi^{2}$ & P-value \\
\hline Number of subjects & 159 & 110 & 49 & & & 126 & 90 & 36 & & \\
\hline Gender (\%female) & 64.1 & 64.5 & 63.3 & 0.024 & $>0.05$ & 61.9 & 61.1 & 63.8 & 0.08 & $>0.05$ \\
\hline Age (mean years \pm s.d.) & $38.17(10.7)$ & $37.9(10.2)$ & $38.8(11.9)$ & & & $38.1(10.3)$ & $38.0(10.2)$ & $38.4(10.7)$ & & \\
\hline Education (mean years \pm s.d.) & $12.5(3.1)$ & $12.8(3.3)$ & $11.9(2.8)$ & & $>0$ & $12.6(3.1)$ & $12.8(3.2)$ & $12.1(2.8)$ & & \\
\hline Scan center $(A / L / G)$ & $42 / 62 / 55$ & $28 / 46 / 36$ & $14 / 16 / 19$ & 1.21 & $>0$. & $24 / 54 / 48$ & $19 / 40 / 31$ & $5 / 14 / 17$ & 1.99 & \\
\hline Diagnosis (MDD/HC) & $118 / 41$ & $84 / 26$ & $35 / 15$ & 0.86 & $>0.05$ & $96 / 30$ & $69 / 21$ & $27 / 9$ & 0.04 & $>0.05$ \\
\hline IDS (mean score \pm s.d.) & $18.90(13.5)$ & $20.06(13.2)$ & $16.27(14.0)$ & & $>0.05$ & $19.46(13.9)$ & $20.05(13.6)$ & $17.91(14.8)$ & & \\
\hline MADRS (mean score \pm s.d.) & $11.89(10.4)$ & $13.01(10.3)$ & $9.39(10.1)$ & & $=0.042$ & $12.78(10.4)$ & $13.53(10.3)$ & $10.80(10.4)$ & & $>0.05$ \\
\hline Duration of SSRI use & $22.91(37.6)$ & $27.77(38.8)$ & $18.80(36.5)$ & & $>0.05$ & $23(36.9)$ & $24.75(37.7)$ & $18.8(36.5)$ & & $>0.05$ \\
\hline SSRI use (no/yes) & $119 / 40$ & $82 / 28$ & $37 / 12$ & & $>0.05$ & $92 / 34$ & $67 / 24$ & $25 / 10$ & & $>0.05$ \\
\hline
\end{tabular}

Abbreviations: HC, healthy controls; IDS, inventory of depressive symptomatology; MADRS, Montgomery-Åsberg Depression Rating Scale; MDD, major depressive disorder; PCLO, piccolo; SSRI, selective serotonin reuptake inhibitor.

Faces System ${ }^{45}$-were chosen for each of five facial expressions (angry, fearful, sad, happy and neutral), comprising 12 female and 12 male faces.

Each particular face was not presented more than four times. The control condition (scrambled faces) was presented 80 times. While functional MRI data were collected, subjects were asked to indicate each face's gender using a button box. During the presentation of scrambled faces, participants had to press left or right buttons in conformity with the instruction presented on the screen, that is, an arrow pointing to the left or to the right. The reaction time (RT) was recorded and used as performance measure.

Executive function paradigm. An event-related parametric version of the ToL task was used to investigate the neural correlates of planning, an aspect of executive functioning. ${ }^{7,34}$ Briefly, while functional MRI data were collected, subjects were asked to work out the minimum number of steps needed-ranging from one to five moves-to get from the start position to the goal position, which were both presented on a screen simultaneously. In the baseline condition, subjects were asked to count the number of yellow and blue beads. RT and proportion of correct answers were used as performance measures.

MRI data acquisition. The functional neuroimaging methods have been comprehensively reported elsewhere. ${ }^{5,7}$ In summary, T2*-weighted echo-planar images sensitive to the blood oxygenation level-dependent (BOLD) effect were acquired using similar Philips 3T MR systems (repetition time $(\mathrm{TR})=2300 \mathrm{~ms}$, echo time $(\mathrm{TE})=30.0 \mathrm{~ms}$ (UMCG: $28.0 \mathrm{~ms}$ ), situated at different locations (Amsterdam, Leiden, and Groningen, the Netherlands). The echo-planar images' volumes were acquired at 35 slices (UMCG: 39 slices), interleaved acquisition, $3 \mathrm{~mm}$ thickness. The matrix sizes were $96 \times 96$ voxels (UMCG: $64 \times 64$ voxels). The in-plane resolution was $2.29 \times 2.29 \mathrm{~mm}^{2}$ (UMCG: $3 \times 3 \mathrm{~mm}^{2}$ ). The images were acquired parallel to the anterior-posterior commissure plane. A T1-weighted anatomical MRI was also acquired for each subject $(\mathrm{TR}=9 \mathrm{~ms}, \mathrm{TE}=3.5 \mathrm{~ms}$, matrix size $256 \times 256$, voxel size $\left.1 \times 1 \times 1 \mathrm{~mm}^{3}\right)$.

\section{Analysis}

Performance. Faces paradigm: RT were analyzed with SPSS (SPSS, Chicago, IL, USA) by means of a repeatedmeasures analysis of covariance (ANCOVA), using RT per valence as dependent factor, group (PCLO +, PCLO-) as between subject factor and age, scan location, and years of education as covariates. Significance for behavioral analyses was set at $5 \%$, and post hoc paired tests were Bonferronicorrected for multiple comparisons. Post hoc analyses were performed to test for interaction using a univariate general linear model.

ToL paradigm: performance data (accuracy and RT) were analyzed with SPSS (SPSS). Performance was analyzed by means of a repeated-measures ANCOVA, using the proportion correct scores and mean response times per trial type as dependent factors, group (PCLO +, PCLO-) as between subject factor, and age as a covariate. Significance for behavioral analyses was set at $5 \%$, and post hoc paired tests were Bonferroni-corrected for multiple comparisons.

Imaging data analysis. Image processing was performed using Statistical Parametric Mapping (SPM; SPM5-http:// www.fil.ion.ucl.ac.uk/spm/) software implemented in Matlab 7.5.0 (The Matlab, Natick, MA, USA). Details of preprocessing and first level single-subject analyses have been described elsewhere. ${ }^{5,7}$ Briefly, following temporal and spatial preprocessing stimulus responses were modeled using delta functions convolved with a synthetic hemodynamic response function. For both paradigms, trial duration was also modeled. ${ }^{46}$ In the faces task, four contrast images were generated for each subject: 'angry $>$ neutral', 'fearful > neutral', 'sad > neutral' and 'happy $>$ neutral'. These contrasts ensured that visual input only differed in its emotional content and were entered in a second-level mixed effect analysis. For the ToL task, activation maps associated with increased task load were calculated per subject, and contrast images were likewise included in a second-level mixed-effect analysis.

Statistical association analysis. The amygdala and ventral striatum were determined a priori as regions of interest (ROI) for the emotional faces task. Positive effects of task across 
whole brain are reported at a threshold of $P<0.05$ familywise error corrected for multiple comparisons $\left(P_{\mathrm{FWE}}<0.05\right)$. We conducted an ANCOVA for the faces task with genotype as an independent factor, to test whether the PCLO risk allele is associated with altered activity in the amygdala during processing of negative faces. Age and scan location were used as covariates. A threshold of $P<0.05$ FWE corrected using the small volume correction $\left(P_{\mathrm{FWE}} \mathrm{svc}<0.05\right)$ procedure implemented in SPM5 for the amygdala was considered significant. For this small volume correction, we used the automated anatomical labeling atlas, ${ }^{47}$ implemented in the Wake Forest University Pick Atlas toolbox. To test whether the $P C L O$ risk allele is associated with altered increased activity in the amygdala and ventral striatum during processing of positive faces, we conducted an ANCOVA with genotype as an independent factor. Age and scan location were used as covariates. A threshold of $P_{\text {FWE_Svc }}<0.05$ (using the automated anatomical labeling atlas, implemented in the Wake Forest University Pick Atlas toolbox) for amygdala and ventral striatum was considered significant.

For the executive function task, regions activated with increasing task load were determined a priori as ROIs according to the areas described in van den Heuvel et al. ${ }^{34}$ (including dorsolateral PFC). Main effects of increasing task load are reported at a threshold of $P<0.05$ whole-brain FWE corrected for multiple comparisons. Next, an ANCOVA using genotype as independent factor was conducted, to test whether the PCLO risk allele is associated with altered brain activity associated with increasing task load. Age, gender, education and scan location were used as covariates. Owing to dorsolateral PFC size, a threshold of $P_{\text {FWE_svc }}<0.05$ $(10 \mathrm{~mm}$ radius around the peak voxel that was found in the main effect of condition) was considered significant.

We conducted post hoc analyses for both tasks to test whether interaction of genotype with current psychopathology occurred. A threshold of $P_{\text {FWE_svc }}<0.05$ (6 $\mathrm{mm}$ radius sphere for amygdala and striatum and $10 \mathrm{~mm}$ radius sphere for cortical areas around the peak voxel) in the ROls was considered significant.

\section{Results}

\section{Processing emotional faces}

PCLO genotype effect on task performance. We did not find significant genotype or genotype $\mathrm{x}$ valence effects on task performance (RT) during emotional face processing.

Additional genotype $x$ diagnosis $x$ valence effects on $R T$ revealed interaction effects between PCLO genotype and MDD diagnosis for angry, fear and sad faces (RT_angry: PCLO*MDD; $P=0.0595 ; \quad R T$ fear: PCLO*MDD; $P=0.0493$; RT_sad: PCLO*MDD; $P=0.0395$; see Supplementary Table 3).

\section{BOLD fMRI results}

Main effect of task during processing of faces. Processing angry faces compared with neutral faces was associated with robustly increased activity of bilateral amygdala as well as bilateral putamen, ventrolateral PFC, superior and middle temporal lobe and inferior parietal cortex. Processing fearful faces compared with neutral faces was similarly associated with increased activity of bilateral amygdala as well as of bilateral parietal cortex, right temporal cortex, bilateral occipital cortex, bilateral amygdala, decreased activity of ventral striatum and right parahippocampal gyrus. Processing sad faces compared with neutral faces activated bilateral amygdala, striatum, insula, parahippocampal area and dorsolateral PFC.

For happy faces, we observed activation in bilateral amygdala and ventral striatum as well as in bilateral occipital cortex, inferior parietal cortex and posterior cingulate cortex.

PCLO genotype effect during processing of faces. We found significantly increased activity in the left amygdala in the $\mathrm{PCLO}+$ group, relative to the PCLO- group, during processing of negative faces (angry vs neutral: (MNI coordinates $x=-21 y=0 z=-15, Z=3.6, P_{\mathrm{FWE} \_s v c}=0.004$, (Figure 1); fear vs neutral: MNI coordinates $x=-18 \quad y=0 \quad z=-18$, $Z=3.24, P_{\text {FWE_svc }}=0.014$, (Figure 2); and trendwise for sad vs neutral: MNI coordinates $x=-15 y=-3 z=-18, Z=2.6$, $P_{\text {FWE_svc }}=0.067$ ).

We did not observe significant effects of PCLO + within our ROls during processing of happy faces. However, we found subtreshold increased activity in the left amygdala during processing of happy faces compared with neutral faces (MNI coordinates $x=-24 \quad y=3 \quad z=-15 ; \quad Z=2.47 ; P_{\mathrm{FWE} \_\mathrm{SVC}}$ $\left.<0.09, P_{\text {uncorrected }}=0.007\right)$. In addition, during processing of happy faces we found a near-significantly decreased activity for the PCLO + group in the right ventral striatum (MNI coordinates $x=9 y=6 z=-9 ; Z=2.67 ; P_{\mathrm{FWE} \_ \text {svc }} \approx$ $\left.0.05(P=0.058) P_{\text {uncorrected }}=0.004\right)$.

We found similar results as reported for negative vs neutral faces, and plotting our data suggested that these effects were indeed driven by negative faces.

Interaction of PCLO genotype with current psychopathology during processing of faces. Post hoc analyses showed a
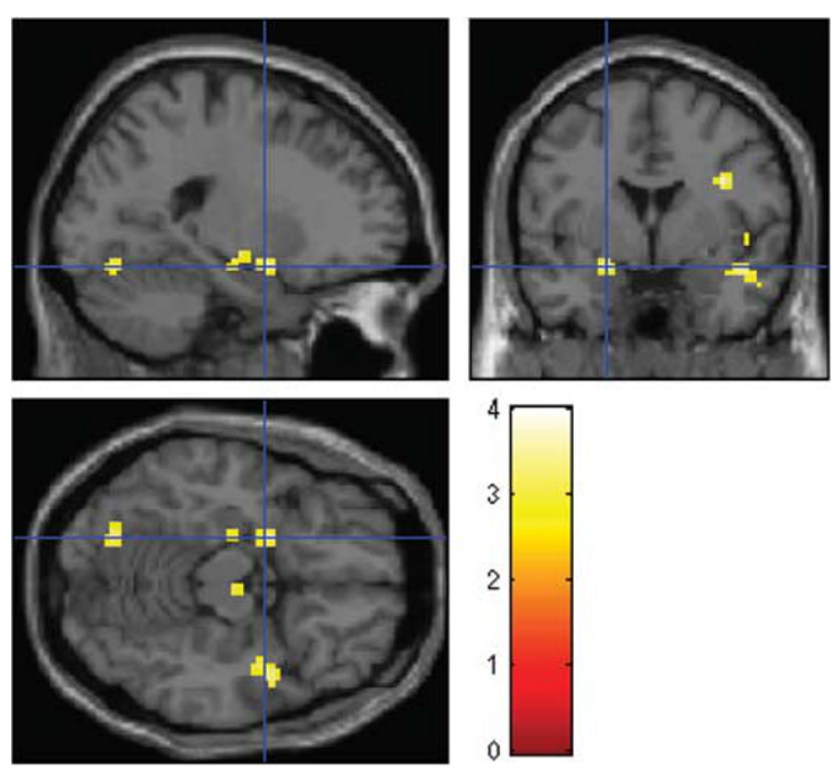

Figure 1 Effect of piccolo (PCLO) genotype on emotion processing of angry vs neutral faces (MNI coordinates $\left.(x=-21 y=0 z=-15), Z=3.6, P_{\mathrm{FWE}}=0.004\right)$. Activation shown at $P<0.005$, uncorrected. 

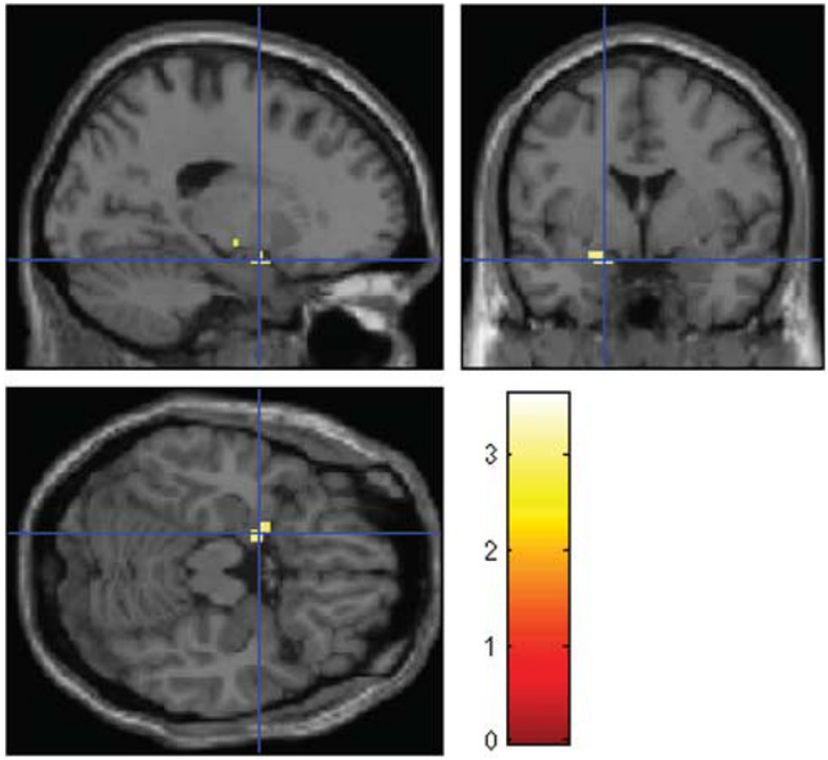

Figure 2 Effect of piccolo (PCLO) genotype on emotion processing of fearful vs neutral faces (MNI coordinates $(x=-18 \quad y=0 \quad z=-18), \quad Z=3.246$, $\left.P_{\mathrm{FWE}}=0.014\right)$. Activation shown at $P<0.005$, uncorrected.

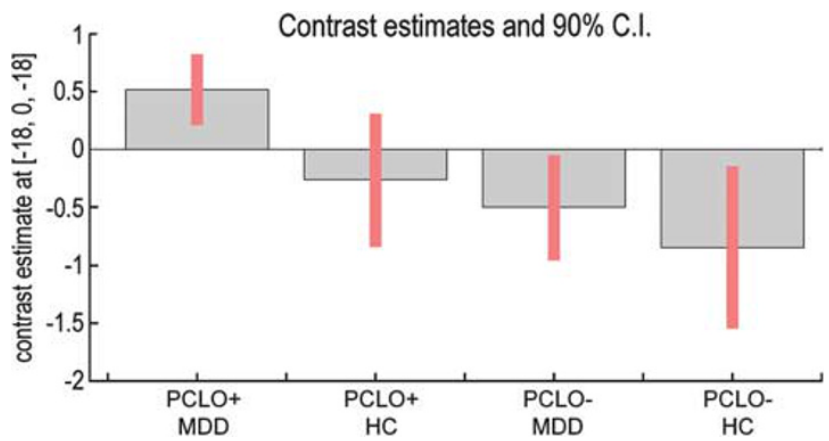

Figure 3 Parameter estimates and 95\% confidence intervals showing direction of interaction effect of genotype and current psychopathology during processing of fearful faces.

significant genotype by depression interaction $(F(1,119)=$ 9.50; $Z=2.8$ ) during processing of fearful faces compared with neutral faces: PCLO + demonstrated increased amygdala activity in the MDD group $\left(Z=3.02 ; p_{\mathrm{FWE}}<0.05\right)$, but not in $\mathrm{HC}(Z=1.1$; Figure 3$)$. There was no interaction during processing of sad or angry faces or during processing of happy faces. Notably, we found no main effect of diagnosis on amygdala activation, which is similar to our previous study. ${ }^{5}$

\section{Executive functioning}

PCLO genotype effect on task performance. No effect of genotype was observed on task performance (mean RT: PCLO+: $8.30 \mathrm{~s} \pm 2.34 ;$ PCLO-: $8.19 \mathrm{~s} \pm 2.11 ; P=0.76$; repeated measures ANOVA for RT per trial type: $P=0.094$; proportion of correct answers: PCLO +: $0.92 \pm$ 0.08 ; PCLO-: $0.90 \pm 0.11 ; P=0.22$; repeated measures
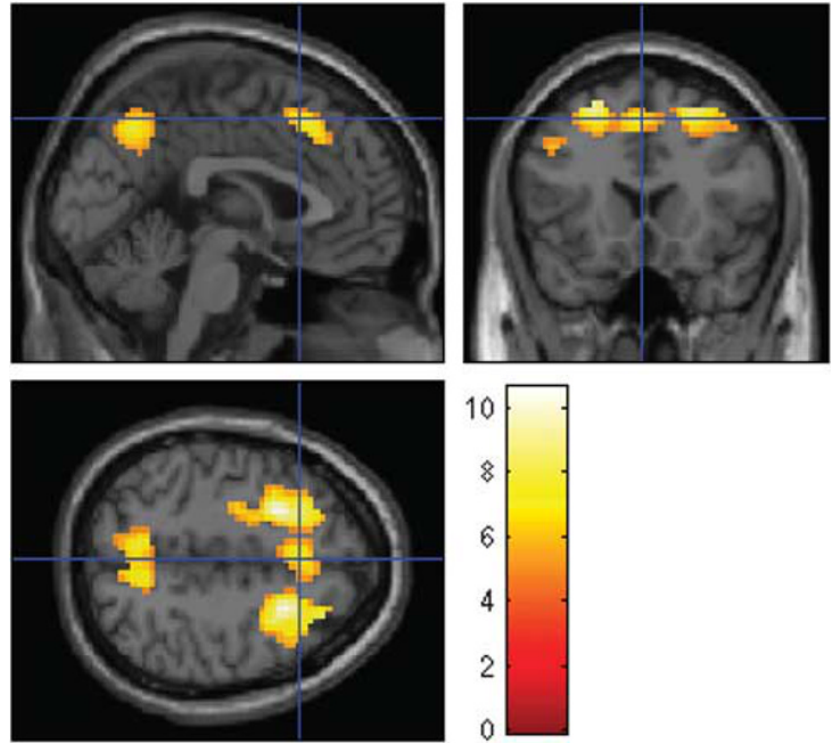

Figure 4 Positive effect of increasing task load on activation during planning. Areas involved in the effect of increasing task load include the dorsolateral prefrontal cortex (PFC), middle PFC, precuneus and inferior parietal lobe. Familywise error corrected at $P<0.05$. Colors indicate $Z$-scores.

ANOVA for proportion of correct answer per trial type: $P=0.488$ )

\section{BOLD fMRI results}

Main effect of task during executive functioning. In accordance with previous studies, ${ }^{7,34}$ increasing task load during the ToL was associated with robust activity in the right dorsolateral PFC, right middle PFC, bilateral precuneus and bilateral inferior parietal lobe (Figure 4; Supplementary Table S2).

PCLO genotype effect during executive functioning. We found no significant effect of genotype in the predefined ROls.

Interaction of PCLO genotype with current psychopathology during executive functioning. Explorative post hoc analyses showed a genotype by diagnosis interaction effect in the left medial dorsolateral PFC (Brodmann's area 9, MNI coordinates $\left.x=-27 y=30 \quad z=24, Z=3.77, P_{\mathrm{FWE}}<0.05\right)$. Increased activity in PCLO + group compared with PCLOwas found in the MDD group but not in $\mathrm{HC}$.

Confounding factors. As is known from other studies, SSRI use may alter regional brain function in psychiatric diseases. ${ }^{48}$ We conducted post hoc analyses for both the emotional face paradigm and the planning paradigm to test for possible effects of SSRI use. During processing of the various emotional faces (angry vs neutral, fear vs neutral, sad vs neutral and happy vs neutral), we found similar results after excluding subjects using SSRIs from the main analyses. Notably, we replicated the effect of PCLO + for the left amygdala. 
Also, post hoc analyses of imaging data of our executive functioning paradigm revealed similar results after excluding subjects using SSRIs as in the main analyses (data not shown).

\section{Discussion}

We report, for the first time and in a large group of subjects, an effect of the PCLO risk allele for MDD on amygdala activity during processing of angry, fearful and (trend wise for) sad facial expression, but not during happy facial processing. During angry and sad facial processing, PCLO risk allele carriers demonstrated increased amygdala activation, independent of psychopathological status. During the processing of fearful faces, only MDD patients with the PCLO risk allele showed increased amygdala activation, whereas no effect of genotype was observed in $\mathrm{HC}$. At a behavioral level, MDD patients with the $P C L O$ risk allele performed near-significantly faster than MDD patients with the nonrisk allele, whereas in $\mathrm{HC}$, no significant differences between PCLO genotype were observed during perception of negative emotions. We found no main effect of genotype on executive function, or on its neural substrate, although explorative analyses indicated MDD-specific effects of $P C L O$ risk allele carriers in the medial prefrontal area.

Dysregulation of the amygdala during emotional information processing has previously been associated with MDD. ${ }^{49-52}$ However, imaging findings have been inconsistent, as several studies failed to demonstrate altered amygdala activity during emotional information processing of faces. ${ }^{5,53-57}$ We suggest that genetic heterogeneity may explain, at least in part, the inconsistencies seen in the literature. Increased amygdala responsiveness during negative face processing and decreased ventral striatum activity in response to positive stimuli have also been reported in MDD studies. Our findings provide further evidence for PCLO being involved in the pathogenetic mechanisms underlying MDD. We found a significant interaction between genotype and current psychopathology, demonstrating increased activity in the amygdala in MDD patients relative to $\mathrm{HC}$ during processing of fearful faces. Involvement of the amygdala in the neural response to fearful faces, but not to angry or sad faces has been suggested by some studies, ${ }^{58-60}$ but not by others. $^{61,62}$

Our study showed lateralization effects in the amygdala in the $P C L O$ risk allele group, which may reflect a bias towards a more specific, sustained emotional reaction in the left amygdala, rather than the general emotional reaction in which the right amygdala is reportedly involved. ${ }^{63}$ It has also been proposed that lateralization may be associated with the temporal dynamics of the amygdala in response to emotional stimuli. Whereas the left amygdala is presumably involved in stimulus evaluation, the right amygdala has been implicated in the rapid detection of emotional stimuli. ${ }^{64}$ Following this line of argument, we suggest that the PCLO variant may modulate evaluation of emotional faces, rather than their rapid detection.

In MDD, treatment with SSRIs may reduce negative bias in perception and memory. ${ }^{65-67}$ In addition, it is thought that changes in serotonergic neurotransmission can modulate identification of emotional faces, particularly fearful faces. ${ }^{65,66}$ Recently, the PCLO risk allele was found to enhance the biological response to SSRIs among MDD patients. ${ }^{23} \mathrm{How}-$ ever, the mechanism of PCLO modulation on serotonin transmission is unclear and may not involve the serotonin transporter, also in view of the inconsistent findings associated with other serotonergic polymorphisms. ${ }^{68,69}$

We found no effect of the PCLO risk allele on performance or BOLD signal during an executive paradigm, the ToL planning task. However, post hoc, we found an interaction between genotype and current psychopathology during performance of the ToL in the left middle dorsolateral PFC, which was also found to be associated with depression severity. ${ }^{7}$ However, these results should be interpreted with care, as these findings were based on explorative analyses. Another potential limitation of our study is that our results cannot be readily generalized outside the field of planning and problem solving, and emotional faces processing field.

Taken together, our findings during emotional face processing and planning indicate that PCLO is associated with emotion processing rather than executive function. Although our findings suggested selectivity of PCLO for serotonergic neurotransmission relative to other monoamines, such a hypothesis is in need of empirical testing. Therefore, future fMRI studies should seek to employ combined cognitiveemotional paradigms coupled with pharmacological manipulation to further investigate the role of PCLO in emotional and cognitive processing.

In conclusion, the PCLO risk allele is associated with increased activity in the left amygdala during emotional information processing of negative faces, but not with executive function or its neural substrate. We suggest that our data support the role of the PCLO risk allele as a risk factor for MDD through altered amygdala activity and, possibly, altered serotonergic neurotransmission in brain areas related to emotional information processing.

\section{Conflict of interest}

S Woudstra, Z Bochdanovits, MJ van Tol, DJ Veltman, FG Zitman, MA van Buchem, EM Opmeer, LR Demenescu, BWJ Penninx declare no conflict of interest. NJA van der Wee received speaking fees from Eli Lilly and Wyeth; and served on advisory panels of Eli LIlly, Pfizer, Wyeth and Servier. A Aleman received an investigator-initiated unrestricted research grant from Brystol-Myers Squibb and speakers bureau honoraria from AstraZeneca, Brystol-Myers Squibb, GlaxoSmithKline. WJ Hoogendijk lectured and advised for, and received unrestricted research grants from Ely Lilli, Lundbeck, Servier, Bristol-Myers Squibb and Organon for which payments were made to the independent research foundation of GGZ ingeest. WJ Hoogendijk holds a patent for geneexpression profiling in peripheral blood as a biomarker for depression. All of these activities are not directly related to the present study and, therefore, do not form a conflict of interest.

Acknowledgements. The infrastructure for the NESDA study (www.nesda.nl) is funded through the Geestkracht program of the Netherlands Organization for Health Research and Development (Zon-Mw, Grant number 10- 
000-1002) and is supported by participating universities and mental health care organizations (VU University Medical Center, GGZ inGeest, Arkin, Leiden University Medical Center, GGZ Rivierduinen, University Medical Center Groningen, Lentis, GGZ Friesland, GGZ Drenthe, Scientific Institute for Quality of Healthcare (IQ healthcare), Netherlands Institute for Health Services Research (NIVEL) and Netherlands Institute of Mental Health and Addiction (Trimbos Institute).

1. World Health Organization (WHO). Mental Health: Facing the Challenges, Building Solutions: report from the WHO European Ministerial Conference of Helsinki. World Health Organization (WHO), 2005

2. World Health Organization (WHO). Global Health Risks-Mortality and Burden of Disease Attributable to Selected Major Risks. World Health Organization (WHO), 28 October 2009.

3. American Psychiatric Association. Diagnostic and Statistical Manual of Mental Disorders, $D S M-I V$, 4th edn, 4th text revision. American Psychiatric Association: Washington, DC 2000

4. Weiner IB. Assessment Psychology. Handbook of Psychology, vol. 10. Wiley: New York, 2003, pp 279-302 (ISBN: 978-0-471-38407-6).

5. Demenescu LR, Renken R, Kortekaas R, van Tol MJ, Marsman JB, van Buchem MA et al. Neural correlates of perception of emotional facial expressions in out-patients with mild-tomoderate depression and anxiety. A multicenter fMRI study. Psychol Med 2011; 41: 2253-2264.

6. Savitz JB, Drevets WC. Imaging phenotypes of major depressive disorder: genetic correlates. Neuroscience 2009; 164: 300-330.

7. van Tol MJ, van der Wee NJ, Demenescu LR, Nielen MM, Aleman A, Renken R et al. Functional MRI correlates of visuospatial planning in out-patient depression and anxiety. Acta Psychiatr Scand 2011; 124: 273-284..

8. Sullivan PF, Neale MC, Kendler KS. Genetic Epidemiology of Major Depression: Review and Meta-Analysis. Am J Psychiatry 2000; 157: 1552-1562.

9. Dannlowski U, Ohrmann P, Bauer J, Kugel H, Baune BT, Hohoff C et al. Serotonergic genes modulate amygdala activity in major depression. Genes, Brain Behav 2007; 6: 672 676.

10. Dannlowski U, Ohrmann P, Bauer J, Deckert J, Hohoff C, Kugel H et al. 5-HTTLPR Biases Amygdala Activity in Response to Masked Facial Expressions in Major Depression. Neuropsychopharmacology 2007; 33: 418-424

11. Heinz A, Braus DF, Smolka MN, Wrase J, Puls I, Hermann D et al. Amygdala-prefronta coupling depends on a genetic variation of the serotonin transporter. Nat Neurosci 2005; 8 : 20-21

12. Lau JY, Goldman D, Buzas B, Fromm SJ, Guyer AE, Hodgkinson C et al. Amygdala function and 5-HTT gene variants in adolescent anxiety and major depressive disorder. Biol Psychiatry 2009; 65: 349-355

13. Lee BT, Ham BJ. Monoamine oxidase A-uVNTR genotype affects limbic brain activity in response to affective facial stimuli. Neuroreport 2008; 19: 515-519.

14. Zill P, Baghai TC, Zwanzger P, Schule C, Eser D, Rupprecht R et al. SNP and haplotype analysis of a novel tryptophan hydroxylase isoform (TPH2) gene provide evidence for association with major depression. Mol Psychiatry 2004; 9: 1030-1036.

15. Bosker FJ, Hartman CA, Nolte IM, Prins BP, Terpstra P, Posthuma D et al. Poor replication of candidate genes for major depressive disorder using genome-wide association data. $\mathrm{Mol}$ Psychiatry 2011; 16: 516-532.

16. Sullivan PF, de Geus EJC, Willemsen G, James MR, Smit JH, Zandbelt T et al. Genomewide association for major depressive disorder: a possible role for the presynaptic protein piccolo. Mol Psychiatry 2009; 14: 359-375.

17. Bochdanovits Z, Verhage M, Smit AB, Geus EJC, Posthuma D, Boomsma DI et al. Joint reanalysis of 29 correlated SNPs supports the role of PCLO/Piccolo as a causal risk facto for major depressive disorder. Mol Psychiatry 2009; 14: 650-652.

18. Hek K, Mulder CL, Luijendijk HJ, van Duijn CM, Hofman A, Uitterlinden AG et al. The PCLO gene and depressive disorders: replication in a population-based study. Hum Mol Genet 2010; 19: 731-734.

19. Shyn SI, Shi J, Kraft JB, Potash JB, Knowles JA, Weissman MM et al. Novel loci for majo depression identified by genome-wide association study of Sequenced Treatmen Alternatives to Relieve Depression and meta-analysis of three studies. Mol Psychiatry 2011; 16: 202-215.

20. Wray NR, Pergadia ML, Blackwood DH, Penninx BW, Gordon SD, Nyholt DR et al. Genome-wide association study of major depressive disorder: new results, meta-analysis, and lessons learned. Mol Psychiatry 2012; 17: 36-48.

21. Betts MJ, Russell RB. Amino acid properties and consequences of substitutions. In: Bioinformatics for Geneticists; MR Barnes, IC Gray (eds). John Wiley \& Sons, Ltd: Chichester, UK, 2003; doi:10.1002/0470867302.ch14.

22. Südhof TC. Neurotransmitter Release. In: Südhof TC, Starke K (eds). Pharmacology of Neurotransmitter Release. 184 edn. Springer: Berlin Heidelberg, 2008, pp 1-21.

23. Schuhmacher A, Mossner R, Hofels S, Pfeiffer U, Guttenthaler V, Wagner M et al. PCLO rs2522833 modulates HPA system response to antidepressant treatment in major depressive disorder. Int J Neuropsychopharmacol 2011; 14: 237-245.

24. Robbins TW, Arnsten AFT. The Neuropsychopharmacology of Fronto-Executive Function: Monoaminergic Modulation. Annu Rev Neurosci 2009; 32: 267-287.
25. Surguladze SA, Young AW, Senior $\mathrm{C}, \mathrm{Br}+\mathbb{R}$ bion G, Travis MJ, Phillips ML. Recognition Accuracy and Response Bias to Happy and Sad Facial Expressions in Patients With Major Depression. Neuropsychology 2004; 18: 212-218.

26. Fusar-Poli $P$, Placentino A, Carletti F, Landi P, Allen $P$, Surguladze $S$ et al. Functional atlas of emotional faces processing: a voxel-based meta-analysis of 105 functional magnetic resonance imaging studies. J Psychiatry Neurosci 2009; 34: 418-432.

27. Daly E, Deeley Q, Hallahan B, Craig M, Brammer M, Lamar M et al. Effects of acute tryptophan depletion on neural processing of facial expressions of emotion in humans. Psychopharmacology 2010; 210: 499-510.

28. Ruhé HG, van Rooijen G, Spijker J, Peeters FPML, Schene AH. Staging methods for treatment resistant depression: a systematic review. J Affect Disord 2012; 137 : 35-45.

29. Fitzgerald Sr PB. An fMRI study of prefrontal brain activation during multiple tasks in patients with major depressive disorder. Hum Brain Mapp 2008; 29: 490-501.

30. McClintock SM, Husain MM, Greer TL, Cullum CM. Association between depression severity and neurocognitive function in major depressive disorder: A review and synthesis. Neuropsychology 2010; 24: 9-34.

31. Goethals I, Audenaert K, Jacobs F, Van de Wiele C, Ham H, Pyck H et al. Blunted prefrontal perfusion in depressed patients performing the Tower of London task. Psychiatry Res 2005; 139: 31-40.

32. Matsuo K, Glahn DC, Peluso MAM, Hatch JP, Monkul ES, Najt P et al. Prefrontal hyperactivation during working memory task in untreated individuals with major depressive disorder. Mol Psychiatry 2006; 12: 158-166.

33. Rose EJ, Simonotto E, Ebmeier KP. Limbic over-activity in depression during preserved performance on the n-back task. Neurolmage 2006; 29: 203-215.

34. van den Heuvel OA, Groenewegen HJ, Barkhof F, Lazeron RHC, van Dyck R, Veltman DJ. Frontostriatal system in planning complexity: a parametric functional magnetic resonance version of tower of london task. Neurolmage 2003; 18: 367-374.

35. Amat J, Baratta MV, Paul E, Bland ST, Watkins LR, Maier SF. Medial prefrontal cortex determines how stressor controllability affects behavior and dorsal raphe nucleus. Nat Neurosci 2005; 8: 365-371.

36. Giorgi O, Lecca D, Piras G, Driscoll P, Corda MG. Dissociation between mesocortical dopamine release and fear-related behaviours in two psychogenetically selected lines of rats that differ in coping strategies to aversive conditions. Eu J Neurosci 2003; 17: 2716-2726.

37. Friedel E, Schlagenhauf F, Sterzer P, Park SQ, Bermpohl F, Strohle A et al. 5-HTT genotype effect on prefrontal-amygdala coupling differs between major depression and controls. Psychopharmacology 2009; 205: 261-271.

38. Lee BT, Lee HY, Lee BC, Pae CU, Yoon BJ, Ryu SG et al. Impact of the tryptophan hydroxylase 1 gene $\mathrm{A} 218 \mathrm{C}$ polymorphism on amygdala activity in response to affective facial stimuli in patients with major depressive disorder. Genes Brain Behav 2009; 8 : 512-518.

39. Penninx BWJH, Beekman AT, Smit JH, Zitman FG, Nolen WA, Spinhoven $P$ et al. The Netherlands Study of Depression and Anxiety (NESDA): rationale, objectives and methods. Int J Methods Psychiatr Res 2008; 17: 121-140.

40. Boomsma DI, Willemsen G, Sullivan PF, Heutink P, Meijer P, Sondervan D et al. Genomewide association of major depression: description of samples for the GAIN Major Depressive Disorder Study: NTR and NESDA biobank projects. Eur J Hum Genet 2008; 16: $335-342$

41. van Tol MJ, van der Wee NJA, van den Heuvel OA, Nielen MMA, Demenescu LR, Aleman A et al. Regional Brain Volume in Depression and Anxiety Disorders. Arch Gen Psychiatry 2010; 67: 1002-1011.

42. Rush AJ, Giles DE, Schlesser MA, Fulton CL, Weissenburger J, Burns C. The Inventory for Depressive Symptomatology (IDS): preliminary findings. Psychiatry Res 1986; 18: $65-87$

43. Montgomery SA, Asberg M. A new depression scale designed to be sensitive to change. Br J Psychiatry 1979; 134: 382-389.

44. Wolfensberger SPA, Veltman DJ, Hoogendijk WJG, Boomsma DI, de Geus EJC. Amygdala responses to emotional faces in twins discordant or concordant for the risk for anxiety and depression. Neurolmage 2008; 41: 544-552.

45. Lundqvist D, Flykt A, Ohmann A. The Karolinska Directed Emotional Faces (KDEF). Karolinska Institute: Stockholm, 1998.

46. Friston KJ, Fletcher P, Josephs O, Holmes A, Rugg MD, Turner R. Event-Related fMRI: Characterizing Differential Responses. Neurolmage 1998; 7: 30-40.

47. Maldjian JA, Laurienti PJ, Kraft RA, Burdette JH. An automated method for neuroanatomic and cytoarchitectonic atlas-based interrogation of fMRI data sets. Neurolmage 2003; 19: 1233-1239.

48. Linden DE. How psychotherapy changes the brain-the contribution of functional neuroimaging. Mol Psychiatry 2006; 11: 528-538.

49. Drevets WC. Neuroimaging Abnormalities in the Amygdala in Mood Disorders. Ann N Y Acad Sci 2003; 985: 420-444.

50. Fu CHY, Williams SCR, Cleare AJ, Brammer MJ, Walsh ND, Kim J et al. Attenuation of the neural response to sad faces in major depression by antidepressant treatment: A prospective, event-related functional magnetic resonance imaging study. Arch Gen Psychiatry 2004; 61: 877-889.

51. Leppanen JM. Emotional information processing in mood disorders: a review of behavioral and neuroimaging findings. Curr Opin Psychiatry 2006; 19: 34-39. 
52. Sheline YI, Barch DM, Donnelly JM, Ollinger JM, Snyder AZ, Mintun MA. Increased amygdala response to masked emotional faces in depressed subjects resolves with antidepressant treatment: an fMRI study. Biol Psychiatry 2001; 50: 651-658.

53. Almeida JRC, Versace A, Hassel S, Kupfer DJ, Phillips ML. Elevated amygdala activity to sad facial expressions: A state marker of bipolar but not unipolar depression. Biol Psychiatry 2010; 67: 414-421.

54. Gotlib IH, Sivers H, Gabrieli JDE, Whitfield-Gabrieli S, Goldin P, Minor KL et al. Subgenual anterior cingulate activation to valenced emotional stimuli in major depression. Neuroreport 2005; 16: 1731-1734

55. Lawrence NS, Williams AM, Surguladze S, Giampietro V, Brammer MJ, Andrew C et al. Subcortical and ventral prefrontal cortical neural responses to facial expressions distinguish patients with bipolar disorder and major depression. Biol Psychiatry 2004; 55: 578-587.

56. Lee BT, Seok JH, Lee BC, Cho SW, Yoon BJ, Lee KU et al. Neural correlates of affective processing in response to sad and angry facial stimuli in patients with major depressive disorder. Prog Neuro-Psychopharmacol Biol Psychiatry 2008; 32: 778-785.

57. Norbury R, Selvaraj S, Taylor MJ, Harmer C, Cowen PJ. Increased neural response to fear in patients recovered from depression: a $3 \mathrm{~T}$ functional magnetic resonance imaging study. Psychol Med 2010; 40: 425-432.

58. Adolphs R, Tranel D, Damasio H, Damasio A. Impaired recognition of emotion in facial expressions following bilateral damage to the human amygdala. Nature 1994; 372: 669-672.

59. Morris JS, Frith CD, Perrett DI, Rowland D, Young AW, Calder AJ et al. A differential neural response in the human amygdala to fearful and happy facial expressions. Nature 1996; 383: 812-815.

60. Thomas EJ, Elliott R, McKie S, Arnone D, Downey D, Juhasz G et al. Interaction between a history of depression and rumination on neural response to emotional faces. Psychol Med 2011; 41: 1845-1855.

61. Fitzgerald DA, Angstadt M, Jelsone LM, Nathan PJ, Phan KL. Beyond threat: Amygdala reactivity across multiple expressions of facial affect. Neurolmage 2006; 30: 1441-1448.
62. Sander D, Grafman J, Zalla T. The human amygdala: an evolved system for relevance detection. Rev Neurosci 2003; 14: 303-316.

63. Baas D, Aleman A, Kahn RS. Lateralization of amygdala activation: a systematic review of functional neuroimaging studies. Brain Res Rev 2004; 45: 96-103.

64. Sergerie K, Chochol C, Armony JL. The role of the amygdala in emotional processing: A quantitative meta-analysis of functional neuroimaging studies. Neurosci Biobehav Rev 2008; 32: 811-830.

65. Alves-Neto WC, Guapo VG, Graeff FG, Deakin JFW, Del-Ben CM. Effect of escitalopram on the processing of emotional faces. Braz J Med Biol Res 2010; 43: 285-289.

66. Del-Ben CM, Ferreira CAQ, Alves-Neto WC, Graeff FG. Serotonergic modulation of faceemotion recognition. Braz J Med Biol Res 2008; 41: 263-269.

67. Harmer CJ, Bhagwagar Z, Perrett DI, Vollm BA, Cowen PJ, Goodwin GM. Acute SSR Administration Affects the Processing of Social Cues in Healthy Volunteers Neuropsychopharmacology 2002; 28: 148-152.

68. Holmes AJ, Bogdan R, Pizzagalli DA. Serotonin transporter genotype and action monitoring dysfunction: A possible substrate underlying increased vulnerability to depression. Neuropsychopharmacology 2010; 35: 1186-1197.

69. Lewis G, Mulligan J, Wiles N, Cowen P, Craddock N, Ikeda M et al. Polymorphism of the 5$\mathrm{HT}$ transporter and response to antidepressants: randomised controlled trial. $\mathrm{Br} \mathrm{J}$ Psychiatry 2011; 198: 464-471.

Translational Psychiatry is an open-access journal published by Nature Publishing Group. This work is licensed under the Creative Commons Attribution-Noncommercial-No Derivative Works 3.0 Unported License. To view a copy of this license, visit http://creativecommons.org/licenses/by-nc-nd/3.0/

Supplementary Information accompanies the paper on the Translational Psychiatry website (http://www.nature.com/tp) 